\title{
Corrigendum
}

\section{The effects of synbiotic supplementation on markers of insulin metabolism and lipid profiles in gestational diabetes: a randomized, double-blind, placebo-controlled trial - CORRIGENDUM}

Shahnaz Ahmadi, Mehri Jamilian, Maryam Tajabadi-Ebrahimi, Parvaneh Jafari and Zatollah Asemi

(First published online 29 September 2016)

DOI: https://doi.org/10.1017/S0007114516003457

\section{Original text and correction}

\section{Original text}

In the present study, 70 GDM women aged 18-40 years without prior diabetes that have been diagnosed with GDM by 'one-step' 2-h 75-g oral glucose tolerance test (OGTT) at 24-28 weeks' gestation referred to Kosar Clinic in Arak, Iran from March 2016 to May 2016 were selected as our patients.

\section{Correction}

The subjects invited from February 20, 2016 till March 19, 2016 from our GDM Referral center in order to investigate the eligibility and recruit the participants. Immediately after recruitment, we started the intervention and our last participant completed the trial on May $3^{\text {rd }} 2016$. Therefore, we completed 6 weeks of intervention for each participant. In the present study, 70 women aged $18-40$ years that had been diagnosed with GDM by 'one-step' 2-h 75-g oral glucose tolerance test (OGTT) at 24-28 weeks' gestation, referred to Kosar Clinic in Arak, Iran, were included. All these data are available in the Iranian website for registration of clinical trials (http://www.irct.ir: IRCT201605085623N77). 